\section{Effects of physical training on sarcomere lengths and muscle-tendon interface of the cervical region in an experimental model of menopause}

\author{
Carolina dos Santos Jacob, ${ }^{1}$ \\ Lara Caetano Rocha, ${ }^{1}$ \\ Jurandyr Pimentel Neto, ${ }^{1}$ \\ Ii-sei Watanabe, ${ }^{2}$ \\ Adriano Polican Ciena ${ }^{1}$
}

'Department of Physical Education, Laboratory of Morphology and Physical Activity, São Paulo State University "Julio de Mesquita Filho", Rio Claro; ${ }^{2}$ Department of Anatomy, Institute of Biomedical Sciences, University of São Paulo, Brazil

\begin{abstract}
The aim of this study was to describe the structural and ultrastructural aspects of the myotendinous junction (MTJ) and the proximal and distal sarcomeres of the sternomastoid of aged Wistar rats subjected to an experimental model of menopause and swimming training. A total of 20 female elderly rats were divided into the following four groups ( $\mathrm{n}=5$ in each group): sedentary/no-menopausal (SNM), trained/nomenopausal (TNM), sedentary/menopausal (SM), and trained/menopausal (TM). The MTJ samples were dissected and analyzed using transmission electron microscopy. We showed that the TNM Group rats exhibited changes in morphological characteristics as a consequence of physical exercise, which included an increase of $36.60 \%(\mathrm{P}<0.001)$ in the evagination length of the MTJ and a reduction in the length of the distal $(77.38 \%) \quad(\mathrm{P}<0.0001) \quad$ and proximal $(68.15 \%)(\mathrm{P}<0.0001)$ sarcomeres. The $\mathrm{SM}$ Group exhibited a reduction of about $275.93 \% \quad(\mathrm{P}<0.001)$ in the muscle-tendon interface and in the lengths of distal sarcomeres $(55.87 \%) \quad(\mathrm{P}<0.0001)$ compared with SNM Group. Our results suggest that the swimming training under experimental model of menopause promoted tissue reorganization and increased muscle-tendon interaction with a drastic development in the length and thickness of the sarcoplasmatic invaginations and evaginations. In addition, the sarcomeres exhibited different lengths and a reduction in both groups subjected to swimming training.
\end{abstract}

\section{Introduction}

The sarcomere presents a series of light and dark bands, where $\mathrm{Z}$ lines are dark, rectilinear, and perpendicular to the myofibrils and the length of each sarcomere is determined between a pair of adjacent $\mathrm{Z}$ lines. ${ }^{1}$ Considering its functionality, the sarcomeres follow a single force-length relationship, and differences in their lengths can result in changes in force after the activation of the muscle fibers. ${ }^{2}$ In addition to evidences that suggests variations in lengths of sarcomeres and nonuniformity in relation to their different locations in the muscle. ${ }^{3}$ Each muscle has distinct morphologies in the face of different levels of contractile strength that they may be subjected to according to the execution of various training protocols, with respect to the angle of the myofibrils and the directions of the collagen fibers of the tendon. ${ }^{4}$

The myotendinous junction (MTJ) is an interface area between the muscle and tendon, which receives the highest transmission of the contractile force of locomotor apparatus, also storage of elastic energy, stability, and position of joints. ${ }^{5}$ Therefore, it is a region highly predisposed to injuries resulting from physical exercise to a sedentary lifestyle in different age groups during excessive efforts and eccentric contractions. ${ }^{6}$

Regular practice of aerobic physical exercise has been denoted as a nonpharmacological intervention with benefits in terms of physiological, functional, and structural aspects of the human body. Swimming is a widely recommended aerobic exercise for middle-aged women, used as a therapeutic modality in the treatment and prevention of diseases and injuries associated with musculoskeletal disorders in aging that demonstrated positive results due to their low impact nature, hydrostatic pressure and water temperature. ${ }^{7,8}$ Furthermore, this physical exercise works to the attenuation of muscular oxidative capacity, which delays the onset of sarcopenia and stimulates the synthesis of proteins and mitochondrial contents to increase metabolic capacity. ${ }^{9-11}$ As a result of several changes in the female organism related to aging, the muscular system suffers multiple morphological changes in this process. Thus, the MTJ area suffers several implications in their morphological and ultrastructural characteristics, causing drastic changes in the transmission of force between the muscle and tendon. If there is no intervention, this process may lead to greater weakness, difficulty to perform activities of daily living, dependence, and in extreme cases, the death of the elderly particularly in women. ${ }^{12}$ For the purpose of investigating and under-
Correspondence: Adriano Polican Ciena, Laboratory of Morphology and Physical Activity, Institute of Biosciences (IB), São Paulo State University "Julio de Mesquita Filho", Rio Claro, SP, Brazil.

Tel. +55.19.3526-4346.

E-mail: adriano.ciena@unesp.br

Key words: Sarcomere; myotendinous junction; menopause model; aging; physical exercise.

Contributions: the authors contributed equally.

Conflict of interest: the authors declare no potential conflict of interest.

Funding: the work was supported by São Paulo Research Foundation (Process: 2017/21764-0 and 2017/12525-1).

Received for publication: 22 April 2019.

Accepted for publication: 5 July 2019.

This work is licensed under a Creative Commons Attribution-NonCommercial 4.0 International License (CC BY-NC 4.0).

(C) Copyright: the Author(s), 2019

Licensee PAGEPress, Italy

European Journal of Histochemistry 2019; 63:3038 doi:10.4081/ejh.2019.3038

standing the morphofunctional changes and mechanisms involved, this phase is simulated by an experimental model denominated ovariectomy, a technique of bilateral ovarian removal, in order to replicate the signs, the symptoms and physiological changes under the female organism. ${ }^{13}$

Therefore, the aim of this study was to describe the adaptations to the lengths of sarcomeres and the associated ultrastructural aspects of the muscle-tendon interface of the sternomastoid of aged female Wistar rats subjected to an experimental model of menopause and swimming training. We hypothesized that it is in this primary region of force transmission that a reduction occurs in the lengths of sarcomeres.

\section{Materials and Methods}

\section{Animals}

A total of 20 female Wistar rats aged 1 year and 2 months were randomly divided into the following four groups $(\mathrm{n}=5$ in each group): sedentary/no-menopausal (SNM): animals not subjected to swimming training and ovariectomy; trained/no-menopausal (TNM): animals subjected to swimming training; sedentary/menopausal (SM): animals subjected to ovariectomy; and trained/menopausal (TM): animals subject- 
ed to ovariectomy and swimming training. The animals were allocated in cages $(33 \times 40 \times 16 \mathrm{~cm})$ with four rats within, under the conditions of temperature monitoring $\left(23 \pm 2^{\circ} \mathrm{C}\right)$ and $12 \mathrm{~h}$ light/dark period, with food and water available ad libitum. The procedures applied in this study were approved by the Committee on Ethics in Animal Use of the Institute of Biosciences of the Paulista State University (no. 1078/2018).

\section{Menopause experimental model}

The SM and TM Groups were anesthetized using ketamine $(50 \mathrm{mg} / \mathrm{kg}$ ) and xylazine $(10 \mathrm{mg} / \mathrm{kg})$ via the intraperitoneal route and subjected to trichotomy and asepsis of the ventral region. The abdominal wall was sectioned for exposure and dissection of the ovaries bilaterally. After this procedure, the rats were subjected to asepsis at the surgical incision site and treated with analgesics (Paracetamol ${ }^{\circledR} 300 \mathrm{mg} / \mathrm{kg}$ ) via drinking water for 7 days postoperatively. ${ }^{14}$

\section{Swimming training protocol}

In the fourth postoperative month, the TNM and TM Groups were subjected to adaptation in a water tank for a week. The swimming training protocol consisted of 5 weekly sessions with duration of $60 \mathrm{~min}$ each that were performed for 8 weeks, com- prising a total of 40 sessions. The animals were placed individually in rectangular tanks containing water heated to $31^{\circ} \mathrm{C}$ and immersed to a depth of $40 \mathrm{~cm} .{ }^{15}$ Overload was used for training equivalent to $5 \%$ of the body mass, which was fixed to the thorax, measured, and corrected weekly. ${ }^{16}$

\section{Transmission electron microscopy}

Animals from each experimental group were euthanized using an overdose of anesthetics through intraperitoneal administration of ketamine $(200 \mathrm{mg} / \mathrm{kg})$ and xylazine $(50$ $\mathrm{mg} / \mathrm{kg}$ ). Then, samples of the sternomastoid muscle-tendon interface $\left(3 \mathrm{~mm}^{3}\right)$ were dissected and immersed in $0.1 \mathrm{M}$ sodium cacodylate buffer solution $(\mathrm{pH} 7.4)$ at $4^{\circ} \mathrm{C}$. Subsequently, the samples were post-fixed with $1 \%$ osmium tetroxide solution at $4{ }^{\circ} \mathrm{C}$ and washed with saline solution. The samples were subjected to dehydration in an increasing series of alcohols $(70 \%, 80 \%$, $90 \%$, and $95 \%$ ) and then embedded in resin. Ultrathin sections of $60 \mathrm{~nm}$ were collected in 200-mesh copper screens (Sigma-Aldrich, St. Louis, MO, USA), contrasted with a $4 \%$ uranyl acetate solution, washed in distilled water, contrasted in an aqueous solution of $0.4 \%$ lead citrate for $3 \mathrm{~min}$, and then washed with distilled water. ${ }^{17}$ The grids were examined using Philips Transmission Electron Microscopy (TEM) CM 100.

\section{Morphometric analysis}

For morphometric analysis, the lengths of the distal and proximal sarcomeres and the muscle-tendon interface $(\mathrm{n}=50)$ were measured for each group. A total of 100 measurements of lengths and thicknesses of sarcoplasmatic invaginations and evaginations of the myotendinous interface were made with the help of the ImageJ software ${ }^{\circledR}$. The obtained mean values were statistically evaluated by analysis of variance (two-way ANOVA, followed by Bonferroni post-hoc test) using the Graph Pad 6.0 software ${ }^{\circledR}$. For the delineation of the myotendinous interface, the relationship base (MTJ)/length of the sarcoplasmatic invaginations was adopted as a measure of the myotendinous interface. ${ }^{18}$

\section{Results}

The swimming training protocol applied in the menopause experimental model in this study resulted in morphological and morphometric adaptations throughout the myotendinous interface (Figures 1 and 2) and in sarcomeres of this region (Figure 3) of the sternomastoid muscle.

In the SNM Group, the myotendinous interface showed an adjacent wide area of
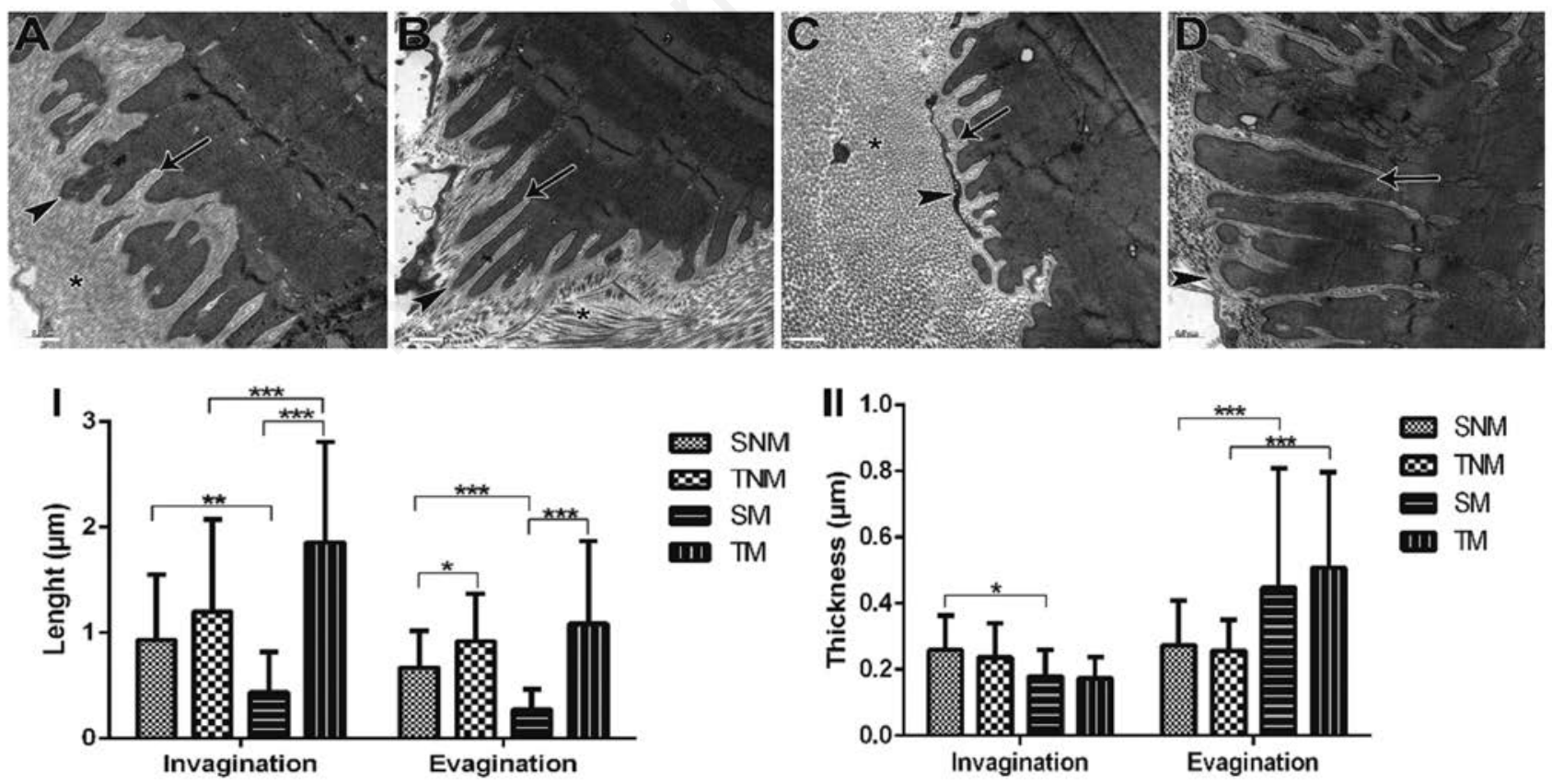

SNM

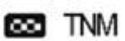

$=\mathrm{SM}$

III TM

Figure 1. A-D) Transmission electron microscopy micrography of the myotendinous junction of the sternomastoid muscle; the organization of the sarcoplasmatic invaginations (arrow), of sarcoplasmatic evaginations (arrow head) and of bundles of collagen fibers (*) is shown; scale bars: $0.5 \mu \mathrm{m}$. I) Mean and standard deviation of length $(\mu \mathrm{m})$ of sarcoplasmatic invaginations and evaginations of the SNM, TNM, SM and TM Groups; ${ }^{* *} \mathrm{P}<0.001 ;{ }^{* * *} \mathrm{P}<0.0001$. II) Mean and standard deviation of thickness ( $\left.\mu \mathrm{m}\right)$ of sarcoplasmatic invaginations and evaginations of the SNM, TNM, SM and TM Groups; ${ }^{*} \mathrm{P}<0.05 ;{ }^{* * *} \mathrm{P}<0.0001$. 
collagen deposition of extracellular matrix. The sarcoplasmatic invaginations from the tendinous tissue were found to be misaligned in parallel to the bundles of myofilaments and communications that extend in proximity to the sarcomere $\mathrm{Z}$ line. Furthermore, the sarcoplasmatic evaginations exhibited arrangements and irregular formats (Figure 1A).

In the TNM Group, large tissue adaptations were observed with various provisions of the bundles of myofilament proteins with the presence of cellular components of tenocytes. The sarcoplasmatic invaginations and evaginations were found to be organized in parallel, especially in the central region of the interface (Figure 1B), There was no significant difference in the sarcoplasmatic invaginations but there was a $29.10 \%$ of increase, and $36.6 \%(\mathrm{P}<0.05)$ in evaginations compared to SNM Group. The thickness of sarcoplasmatic invaginations and evaginations presented no statistical difference between these Groups, but increase of $8.8 \%$ and $5.8 \%$, respectively.

When TNM Group was compared with TM there was decreased by $64.76 \%$ $(\mathrm{P}<0.0001)$ in length of sarcoplasmatic invaginations, while about evaginations there was no difference. Although, the thickness of sarcoplasmatic evaginations increased $97.66 \%(\mathrm{P}<0.0001)$, and invagi- nations $26.58 \%$ but no statistical difference.

The SM Group showed ultrastructural disarrangement in the extension of the myotendinous interface with a wide area of collagen deposition in the tendinous tissue where the cellular fragments of tenocytes spread, and the sarcoplasmatic invaginations derived from the extracellular matrix were extremely short with irregular formats (Figure1C), with reduction of $53.1 \%$ $(\mathrm{P}<0.001)$ in the length of the sarcoplasmatic invagination and $59.3 \%(\mathrm{P}<0.0001)$ of evaginations compared with animals in the SNM Group. Furthermore, the thickness of sarcoplasmatic evagination of SM Group presented increased by $64.10 \%(\mathrm{P}<0.0001)$, and the invagination was increased by $30.76 \%(\mathrm{P}<0.05)$.

Rats in the TM Group exhibited thin collagen fibers present in the entire area of the interface. The sarcoplasmatic invaginations were elongated, thin, with communications, and parallel to the bundles of myofilaments of sarcomeres in series (Figure1D) with an increase of $325.6 \%$ $(\mathrm{P}<0.0001)$, and then in evaginations there was $298.1 \%(\mathrm{P}<0.0001)$ of increased compared to that in the SM Group. There was no difference in thickness of sarcoplasmatic invaginations and evaginations between TM and SM Group, but increase of 3.3\% and $11.81 \%$, respectively. Regarding the length of sarcomeres (Figure 3), we identified the presence of reduced myofilaments, and misalignment of sarcomeres in series in the SM Group with a greater reduction in distal sarcomeres by $55.87 \%(\mathrm{P}<0.0001)$ compared with the SNM Group, and $6.57 \%$ in proximal but no was difference. When the sarcomeres of the SM Group were compared to those of the TM, there was decreased by $36.65 \%(\mathrm{P}<0.001)$ in distal and increased by $155.42 \%(\mathrm{P}<0.0001)$ in proximal. The swimming training protocol

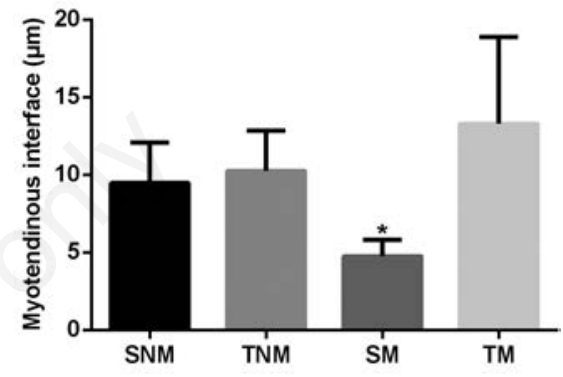

Figure 2. Mean and standard deviation of length $(\mu \mathrm{m})$ of the myotendinous interface of the SNM, TNM, SM and TM Groups; ${ }^{*} \mathrm{P}<0.001$.
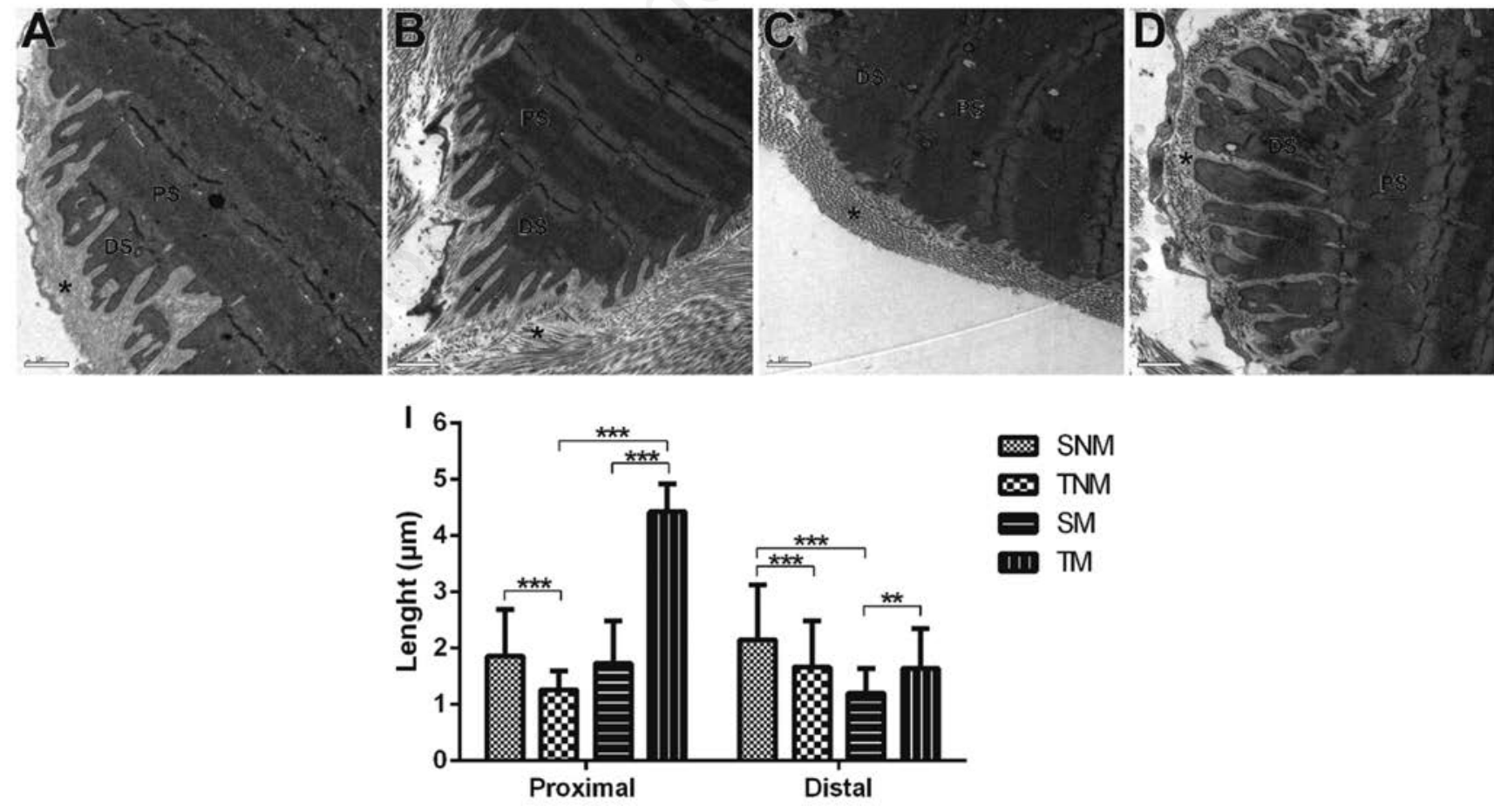

Figure 3. A-D) Transmission electron microscopy micrography of the myotendinous junction of the sternomastoid muscle; the organization of the proximal sarcomere (PS), of the distal sarcomere (DS) and of bundles of collagen fibers $\left(^{*}\right)$ is shown; scale bars: $1 \mu \mathrm{m}$. I) Mean and standard deviation of length $(\mu \mathrm{m})$ of the proximal sarcomere and distal sarcomeres of the SNM, TNM, SM and TM Groups; ${ }_{* *} \mathbf{P}<0.001,{ }^{* * *} \mathbf{P}<0.0001$. 
(TNM Group) resulted in sarcomeres organized in series along the longitudinal axis of the MTJ, delimited by sarcoplasmatic invagination, with decreases of $68.15 \%$ $(\mathrm{P}<0.0001)$ and $77.38 \%(\mathrm{P}<0.0001)$ in the proximal and distal sarcomeres, respectively, compared to those in the SNM Group. Moreover, there was an increase of $250.11 \% \mathrm{P}<0.0001$ ) in proximal sarcomeres and $1.31 \%$ in distal, with no statistical difference, of TM when compared to the TNM Group.

About morphometric analysis of the length of the muscle-tendon interface, the SM Group revealed $179.52 \% \quad \mathrm{P}<0.0001$ ) increased compared to that TM Group (Figure 2).

\section{Discussion}

This study has demonstrated the benefits of swimming training based on the reorganization of the ultrastructural and morphometric aspects of the MTJ and of the sarcomeres of the sternomastoid in aging female rats in the menopause experimental model.

The SNM Group exhibited an extensive area of collagen deposition in the MTJ with irregular and asymmetrical projections, whereas the sarcomeres in this region showed a disarrangement, such that these morphological changes in the muscle tissue and tendon characterize muscle atrophy. Due to physical inactivity, the resulting muscular atrophy leads to a reduction in muscle quality, more muscle weakness, difficulty or inability to perform activities of daily living, dependency, and, in extreme cases, death of the individual with age, particularly women. ${ }^{13}$

The sternomastoid MTJ showed changes resulting from aging associated with a sedentary lifestyle, promoting tissue disruption through the deposition of collagen in the tendinous tissue with thickening near the area of interaction and misalignment and irregular interdigitations. Similar morphological adaptations were reported describing the morphology of the medial pterygoid MTJ of aging rats, which belongs to the masticatory apparatus that inserts into the medial surface of the mandible. ${ }^{19}$

In our study, the lengths of the sarcoplasmatic invaginations and evaginations were higher in the groups subjected to the training protocol. These morphological changes in myotendinous interface suggests an advantageous distribution of the contractile force, thus, a lower level of force can be transferred to each point. ${ }^{20,21}$ In the SM Group that was deprived of ovarian hormones, there was a considerable quantity of fragments of tenocytes that characterized the muscle atrophy. Sarcoplasmatic invaginations and evaginations were extremely reduced, and as a consequence there was shorter length of muscle-tendon interaction. Menopause is generally associated with an increase in adipose tissue and a decrease in muscle mass and strength with or without weight gain. ${ }^{22}$ Some studies have demonstrated the effects of physical exercise under the MTJ, mainly during rehabilitation. Kojima et al. showed that the area of contact between muscle tissue and tendon collagen fibers was reduced by approximately $50 \%$ in both types I and II fibers. ${ }^{23}$ After the swimming training protocol, morphological characteristics of cellular remodeling and restructuring of the MTJ were observed, primarily in the TM Group in the length and thickness of the sarcoplasmatic invaginations and evaginations and in the length of the proximal and distal sarcomeres analyzed in this study. The ramification of the sarcoplasmatic invaginations and evaginations allows the contact areas to increase in size, that leads to the enlargement of the entire tendon-muscle surface area and, therefore, a better resistance to tension. ${ }^{24}$

Aerobic training in women in menopause entails significant benefits in terms of the reduction of body fat and triglyceride levels and loss of muscle mass and fat, which improve muscle quality. ${ }^{25}$ In addition, aerobic exercises at different intensities concomitant with the physiological effects of training have been shown to induce morphological changes in the gastrocnemius MTJ, with an increase in the area of interaction between tissues. ${ }^{26}$

For exerting the extreme ability of tissue plasticity and acting at the interface between the cells of skeletal muscle and tendinous cells, the MTJ exhibits a high level of morphological adaptations, such as an increase in the length and thickness of the sarcoplasmatic invaginations and evaginations resulting from physical exercise, aging, or development according to the function, angle, or topography of skeletal striated muscles. ${ }^{27}$

The ultrastructural units of muscle fibers demonstrated large adjustments in their lengths. Alterations were observed in the lengths of the last (distal) and the penultimate (proximal) sarcomeres present in the MTJ organization, with an emphasis on the distal sarcomeres. We assume that the distal sarcomeres are indicators of the addition of new sarcomeres in series with the formation of the central region to the cell periphery of MTJ. ${ }^{14}$

Sarcomerogenesis, under the conditions of chronic stretching, can be a regenerative process that requires the participation of satellite cells. ${ }^{28}$ The initial length of the sar- comere has an influence on the strength of muscle contraction, and the stiffness of the tendon determines the size of shortening that occurs in this length even when the angle of the linkage does not change. ${ }^{29}$

Through the swimming training protocol, the proximal and distal sarcomere segments exhibited an increase in length, more evident bands I, and alignment in their organization.

This increase in the length of sarcomeres can reduce the spacing between thin and thick filaments, which consequently increases the production of force. ${ }^{30}$ Swimming training has several benefits to several organ systems of the human body and has been used primarily in the treatment of musculoskeletal disorders and prevention of diseases to improve muscle power and strength, flexibility, and agility. ${ }^{31}$

As a result of the experimental design of this initial research project, the variation represented in the results is limited in accordance with the number of samples $(n=5)$. Additional studies should be made in order to more fully examine the adaptations in MTJ in menopause model in front of aerobic training.

The swimming training protocol of sternomastoid muscle under isometric contraction indirect demonstrated its beneficial effects through the morphological adaptations in the muscle-tendon interface in aging allied to menopause in development and in the large changes in MTJ, as the length of the sarcoplasmatic invaginations where such changes are more relevant in training after the ovariectomy. The distal and proximal sarcomeres exhibited different adaptations in their lengths, where in the distal sarcomere lengths were reduced compared to that of the proximal sarcomeres, due to the hormonal deprivation, which supports our hypothesis that the sarcomeres demonstrated different lengths in the region of the MTJ.

\section{References}

1. Velleman SG, Clark DL, Tonniges JR. The Effect of the wooden breast myopathy on sarcomere structure and organization. Avian Dis 2018;62:28-35.

2. Rassier DE. Sarcomere mechanics in striated muscles: from molecules to sarcomeres to cells. Am J Physiol Cell Physiol 2017;313:134-45.

3. Moo EK, Fortuna R, Sibole SC, Abusara Z, Herzog W. In vivo sarcomere lengths and sarcomere elongations are not uniform across an intact muscle. Front Physiol 2016;7:1-9.

4. Secomb JL, Lundgren LE, Farley OR, Tran TT, Nimphius S, Sheppard JM. 
Relationships between lower-body muscle structure and lower-body strength, power, and muscle-tendon complex stiffness. J Strength Cond Res 2015;29:2221-8.

5. Trotter JA. Structure-function considerations of muscle-tendon junctions. Comp Biochem Phys A 2002;133:112733.

6. Kjaer M. Role of extracellular matrix in adaptation of tendon and skeletal muscle to mechanical loading. Physiol Rev 2004;84:649-98.

7. Tanaka H. Swimming exercise: impact of aquatic exercise on cardiovascular health. Sport Med 2009;39:377-87.

8. Mohr M, Helge EW, Petersen LF, Lindenskov A, Weihe P, Mortensen J, et al. Effects of soccer vs swim training on bone formation in sedentary middle aged women. Eur J Appl Physiol 2015;115:2671-9.

9. Menshikova EV, Ritov VB, Liane F, Ferrell RE, Kelley D, Goodpaster BH. Effects of exercise on mitochondrial content and function in aging human skeletal muscle. J Gerontol A-Biol 2006;61:534-40.

10. Landi F, Marzetti E, Martone AM, Bernabei, R, Onder G. Exercise as a remedy for sarcopenia. Curr Opin Clin Nutr 2014;17:25-31.

11. Liu CJ, Chang WP, de Carvalho IA, Savage KEL, Radford LW, Amuthavalli JT. Effects of physical exercise in older adults with reduced physical capacity: meta-analysis of resistance exercise and multimodal exercise. In J Rehab Res 2017;40:1-12.

12. Alexandre TdaS, Duarte YA, Santos JL, Wong R, Lebrão ML. Sarcopenia according to the European Working Group on Sarcopenia in Older People (EWGSOP) versus dynapenia as a risk factor for mortality in the elderly. J Nutr Health Aging 2014;18:751-6.

13. Sophocleous A, Idris AI. Rodent models of osteoporosis. Bonekey Rep 2014; 3:1-9.
14. Sierra LR, Fávaro G, Cerri BR, Rocha LC, de Almeida SRY, Watanabe I, et al. Myotendinous junction plasticity in aged ovariectomized rats submitted to aquatic training. Microsc Res Tech 2018;7:1-8.

15. Pestana PRD, Alves AN, Fernandes KPS, da Silva Junior JA, França CM, Martins MD, et al. [Efeito da natação na expressão de fatores regulatórios miogênicos durante o reparo do músculo esquelético de rato].[Article in Portuguese]. Rev Bras Med Esp 2012;18:419-22.

16. Ciabattari O, Dal Pai A, Dal Pai V. [Efeito da natação associado a diferentes dietas sobre o músculo tibial anterior do rato: estudo morfológico e histoquímico].[Article in Portuguese]. Rev Bras Med Esp 2005;11:121-5.

17. Ciena AP, de Almeida SR, Dias FJ, Bolina CS, Issa JP, Iyomasa MM, et al. Fine structure of myotendinous junction between the anterior belly of the digastric muscle and intermediate tendon in adults rats. Micron 2012;43:258-62.

18. de Palma L, Marinelli M, Pavan M, Bertoni-Freddari C. Involvement of the muscle-tendon junction in skeletal muscle atrophy: an ultrastructural study. Rom J Morphol Embryol 2011;52:105-9.

19. Ciena AP, Luques IU, Dias FJ, Almeida SRY, Iyomasa MM, Watanabe I. Ultrastructure of the myotendinous junction of the medial pterygoid muscle of adult and aged Wistar rats. Micron 2010;41:1011-4.

20. Charvet B, Ruggiero F, Guellec D. The development of the myotendinous junction. A review. Muscles Ligaments Tendons J 2012;2:53-63.

21. Curzi D, Sartini S, Guescini M, Lattanzi D, Di Palma M, Ambrogini P, et al. Effect of different exercise intensities on the myotendinous junction plasticity. PloS One 2016;11:e0158059.

22. Curzi D, Ambrogini P, Falcieri E, Burattini S. Morphogenesis of rat myotendinous junction. Muscles
Ligaments Tendons J 2013;3:275-80.

23. Kojima H, Sakuma E, Mabuchi Y, Mizutani J, Horiuchi O, Wada I, et al. Ultrastructural changes at the myotendinous junction induced by exercise. J Orthop Sci 2008;13:233-9.

24. Curzi D, Salucci S, Marini M, Esposito F, Agnello L, Veicsteinas A, et al. How physical exercise changes rat myotendinous junctions: an ultrastructural study. Eur J Histochem 2012;56:e19.

25. Yeh ML, Liao RW, Hsu CC, Chung YC, Lin JG. Exercises improve body composition, cardiovascular risk factors and bone mineral density for menopausal women: A systematic review and metaanalysis of randomized controlled trials. Appl Nurs Res 2018;40:90-8.

26. Larson RD, Misic MM, Evans E.M. Association of adiposity and muscle quality with physical function differs in young and old women. Menopause 2015;22:337-41.

27. Curzi D. Ultrastructural study of myotendinous junction plasticity: from disuse to exercise. Sport Sci Health 2016;12:279-86.

28. Ciena AP, Almeida SRY, Alves PHM, Bolina-Matos RS, Dias FJ, Issa JPM, et al. Histochemical and ultrastructural changes of sternomastoid muscle in aged wistar rats. Micron 2011;42:871-6.

29. Kinney MC, Dayanidhi PT, Dykstra BS, Mccarthy JJ, Peterson CA, Lieber RL. Reduced skeletal muscle satellite cell number alters muscle morphology after chronic stretch but allows limited serial sarcomere addition. Muscle Nerve 2016;55:384-92.

30. Macintosh BR. Recent developments in understanding the length dependence of contractile response of skeletal muscle. Eur J App Physiol 2017;117:1059-71.

31. Fenwick AJ, Leighton RS, Tanner BC. Myosin mgadp release rate decreases as sarcomere length increases in skinned rat soleus muscle fibers. Biophys J 2016;111:2011-23. 